\title{
Ephemeral third ring of radiation makes appearance around Earth
}

NASA probes observed new Van Allen belt for weeks before solar shock wave wiped it out.

\section{Ron Cowen}

28 February 2013

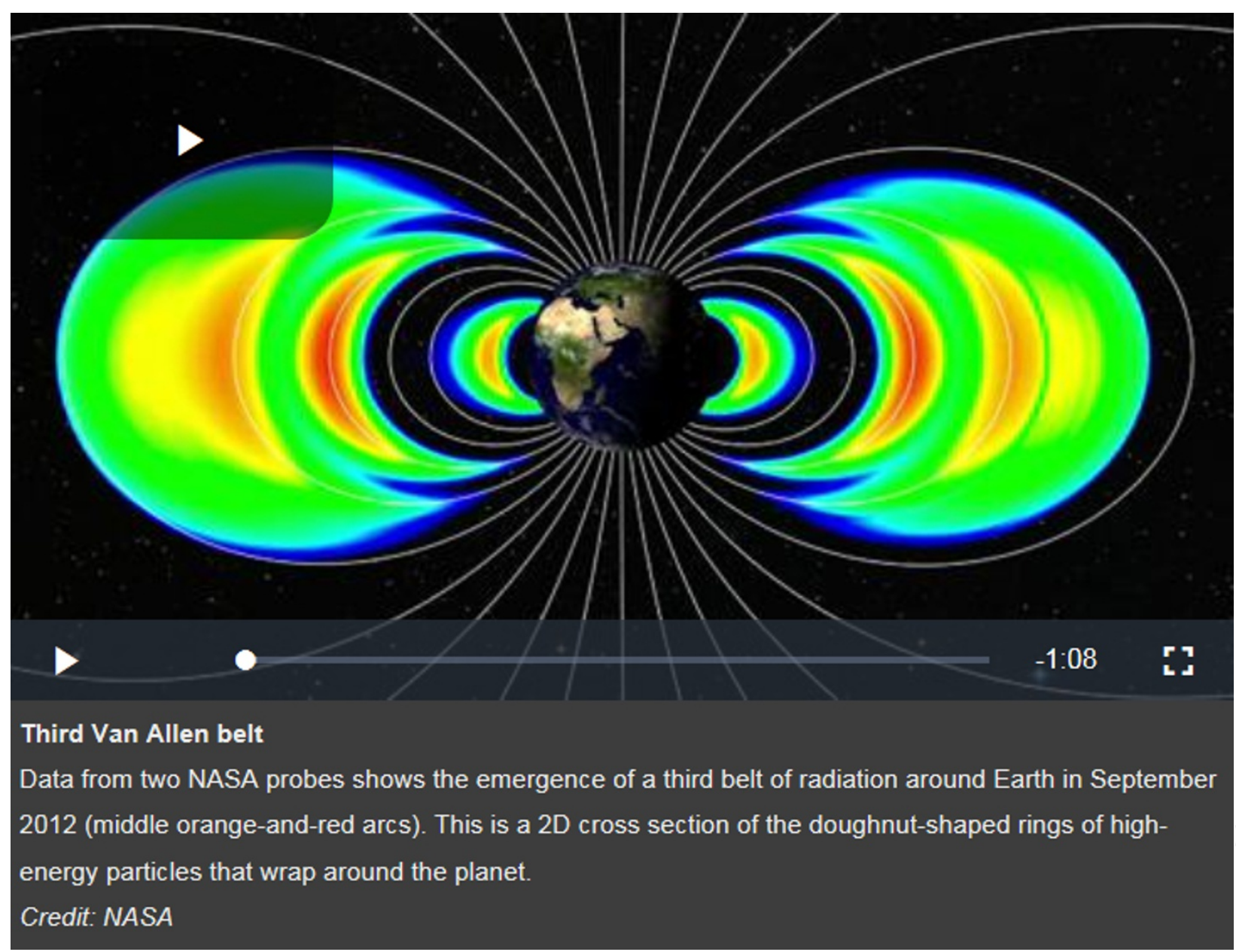

When NASA scientists launched twin spacecraft to probe the Van Allen radiation belts last summer, they were expecting to study two rings of high-energy particles circling Earth. Instead they found three, overturning a 50-year-old model of the giant rings' structure.

First discovered in 1958, the Van Allen belts have been thought to comprise two reservoirs of high-speed, electrically charged particles, corralled into separate doughnut-shaped rings by Earth's magnetic field. The outer ring orbits at a distance of some 10,00060,000 kilometres above Earth, and encircles an inner band of even more energetic particles, roughly 100-10,000 kilometres above Earth. That's the configuration the belts were in when James Van Allen first spotted them using satellite data half a century ago, and that's also the structure that NASA's twin Van Allen Probes recorded when they began operation on 1 September 2012.

But just two days later, telescopes on the probes revealed the emergence of an additional, narrow belt of charged particles sandwiched between the inner ring and a now highly eroded outer ring. "It was so unexpected that we thought there was something wrong with the instrument," says Daniel Baker, a space physicist at the University of Colorado in Boulder.

The new ring persisted, however, and Baker and his team now attributes its creation to an interplanetary shock wave — a travelling outburst of solar-wind particles from the Sun — that has been detected by other craft. The shock wave scoured away much of the outer ring and then divided the remains into two distinct sections, he suggests.

For nearly all of September, the middle ring remained while the outer ring continued to wax and wane. On 1 October, however, another interplanetary shock wave, more energetic than the previous one, obliterated both the outer and middle rings, leaving only the inner Van Allen belt intact. Seven or eight days later, a third shock wave somehow restored the original structure of the two belts as depicted 


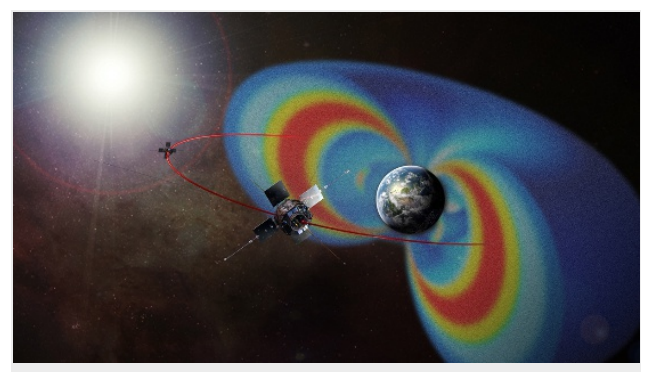

NASA

The two Van Allen Probes orbit through the radiation belts that surround Earth, shown in cross section in this artist's impression.
Baker goes on to say that data collected by the probes on 9 October revealed that "suddenly the whole outer belt was lit up again but with the [middle] ring gone."

The findings, which are reported today in Science ${ }^{1}$, demonstrate that solar outbursts are indeed a strong driving force behind the structure of the belts and that similar activity might be common, especially now, near the peak of the 11-year solar cycle, Baker says. Nonetheless, he adds, details about the dramatic reshaping of the outer belt and the location of the month-long middle ring cannot be explained by current theory.

Understanding the three-zone structure "will be a subject of future research by theorists and numerical modellers all over the world", says Yuri Shprits, a geophysicist at the University of California, Los Angeles, who was not involved with the study.

Nature | doi:10.1038/nature.2013.12529

\section{References}

1. Baker, D. N. et al. Science http://dx.doi.org/10.1126/science.1233518 (2013). 